Matematikai Közlemények

VI. kötet, 2018

doi:10.20312/dim.2018.01

\title{
On connections between domain specific constants in some norm inequalities
}

\author{
Sándor Zsuppán \\ Berzsenyi Dániel Evangélikus (Líceum) Gimnázium és Kollégium \\ zsuppans@gmail.com
}

\begin{abstract}
ÖSSZEFOGLALÓ. Kapcsolatot vezetünk le a divergenciára vonatkozó Babuška-Aziz és a kapcsolódó Friedrichs-Velte egyenlőtlenségben szereplő optimális tartományspecifikus konstansok és a gradiensre vonatkozó kibővített Poincaré egyenlőtlenségben szereplő megfelelő konstans között. Ugyanezzel a módszerrel igazolunk egy új, a rotációra vonatkozó kibővített Poincaré egyenlőtlenséget és kapcsolatát a rotációra vonatkozó Babuška-Aziz egyenlőtlenséggel.
\end{abstract}

\begin{abstract}
We derive connections between optimal domain specific constants figuring in the Friedrichs-Velte inequality for conjugate harmonic functions, in the Babuška-Aziz inequality for the divergence and in the improved Poincaré inequality for the gradient. With the same method we obtain for spatial domains an improved Poincaré inequality for the rotation in connection with the corresponding Babuška-Aziz inequality.
\end{abstract}

\section{Introduction}

In [11] Friedrichs proved an inequality between the norms of square integrable conjugate harmonic functions on planar domains. Horgan and Payne [13] discovered that a smooth simply connected planar domain supports the Friedrichs inequality if and only if it supports the Babuška-Aziz inequality for the divergence [3], which ensures the stable solvability of the divergence equation in an appropriate function space on the domain. Moreover, they proved an important equation involving the optimal domain specific constants figuring in the corresponding inequalities. Velte [21] generalized this connection for smooth simply connected spatial domains and for the Babuška-Aziz inequality for the rotation using another variant of the Friedrichs inequality. Costabel et al. [6] proved that this connection between the Friedrichs-Velte and Babuška-Aziz inequalities and constants remains valid without any smoothness assumptions on the domain and can be further generalized for differential forms on arbitrary dimensional domains, see [7].

These inequalities and constants are not only of theoretical but also of practical interest for the numerical solutions of problems in fluid dynamics and elasticity, see [8,19,20] and references therein. Despite of their importance exact values of these constants are known in a few cases [11,21], altough the inequalities are proved to be valid on general classes of domains. Shapiro [18] proved the Friedrichs inequality for planar domains satisfying an interior cone condition, Acosta et al. [1] established the Babuška-Aziz inequality for the

KULCSSZAVAK. Friedrichs-Velte egyenlőtlenség, Babuska-Aziz egyenlőtlenség, Poincaré egyenlötlenség.

KEYWORDS. riedrichs-Velte inequality, Babuska-Aziz inequality, Poincaré inequality. 
divergence in the class of John domains, which is a generalization of the class of domains satisfying an interior cone condition. Recently Jiang et al. [15] proved that the validity of the Babuška-Aziz inequality is equivalent to the John condition and to the validity of an improved Poincaré inequality [14] provided the domain satisfies the separation property (which condition is fulfilled for any simply connected plane domain).

In this paper we focus rather on the constants than on the inequalities itself. Motivated by a result in [10] we establish a connection between the optimal constant in the improved Poincaré inequality and the Friedrichs-Velte constant, which connection implies the simultaneous validity of the corresponding inequalities. With the same method using the Friedrichs constant connected to Babuška-Aziz constant for the rotation we derive an improved Poincaré inequality using the rotation instead of the gradient.

In section 2 we formulate the notation and the preliminaries. Next in section 3 we derive the main result, Theorem 3.13, which states that domains satisfying the Hardy inequality simultaneously support the Friedrichs-Velte and the improved Poincare inequalities. As a byproduct we obtain upper estimations for the constant in the improved Poincare inequality for star-shaped domains using known upper estimations for the Friedrichs-Velte constants from $[6,13,17]$. We also discuss geometric conditions for the problem domain in order to satisfy the conditions of Theorem 3.13.

\section{Notation and preliminary results}

In this paper $\Omega$ denotes a bounded domain in $\mathbb{R}^{n}$. Let $L_{2}(\Omega)$ be the usual space of square integrable functions over $\Omega$. The norm and the integral mean on $\Omega$ of $f \in L_{2}(\Omega)$ are $\|f\|^{2}=$ $\int_{\Omega}|f|^{2}$ and $f_{\Omega}=\frac{1}{|\Omega|} \int_{\Omega} f$, respectively. $H^{1}(\Omega)$ denotes the Sobolev space of functions with $\nabla f \in L_{2}(\Omega)^{n}$. Its subspace $H_{0}^{1}(\Omega)$ is the closure of smooth functions with compact support in $\Omega$ under the norm $\|f\|_{1}^{2}=\int_{\Omega}|f|^{2}+|\nabla f|^{2}$ of $H^{1}(\Omega) .|f|_{1}=\|\nabla f\|$ denotes the seminorm on $H_{0}^{1}(\Omega)$ equivalent to the $H^{1}$-norm.

Definition 2.1. The domain $\Omega \subset \mathbb{R}^{n}(n=2,3)$ supports the Friedrichs-Velte inequality if there is a positive constant $\Gamma$ depending only on the domain $\Omega$ such that for every pair of square integrable conjugate harmonic functions $u$ and $v$ there holds

$$
\|u\|^{2} \leq \Gamma\|v\|^{2} \text { provided } u_{\Omega}=0 .
$$

The least possible constant in (1) is denoted by $\Gamma_{\Omega}$ and is called the Friedrichs-Velte constants of the domain $\Omega$. Conjugate harmonic means in Definition 2.1. the Cauchy-Riemann equations

$$
\nabla u=\nabla^{\perp} v
$$

with $\nabla^{\perp}=\left(\partial_{2},-\partial_{1}\right)$ for planar domains and the Moisil-Teodorescu equations

$$
\operatorname{rot} v=\nabla u \text { and } \operatorname{div} v=0
$$

for spatial domains. The normalization $u_{\Omega}=0$ means that $u$ belongs to the orthogonal complement of the kernel of the gradient in $L_{2}(\Omega)$. The inequality (1) was investigated by Friedrichs [11] and Shapiro [18] for planar domains and then by Velte, [21] for simply connected spatial domains with sufficiently smooth boundary. Velte [21] also formulated 
another related inequality for simply connected spatial domains estimating the norm of the vector valued function $v$ in (3) as

$$
\|v\|^{2} \leq \tilde{\Gamma}\|u\|^{2} \text { provided } \int_{\Omega} v \cdot \nabla \phi=0 \text { for every } \phi \in H^{1}(\Omega)
$$

This normalization means that the solution $v$ of (3) belongs to the orthogonal complement of the kernel of rot in $L_{2}(\Omega)^{3}$. On simply connected domains it is equivalent to $\operatorname{div} v=0$ in the domain and $v \cdot n=0$ on the boundary where $n$ denotes the unit normal vector.

The exact value of the Friedrichs-Velte constant does not depend on the size of $\Omega$ only on its shape. Its value is known only for a few domains, $[6,13,17]$ contain useful estimations for the class of star-shaped domains. Costabel [7] developed a generalization of the FriedrichsVelte inequality for differential forms which generalization incorporates also the unification of (1) and (4) along with (2) and (3) and it is valid at least in the class of Lipschitz domains.

As observed in $[6,13,21]$ the Friedrichs-Velte inequalities and constants are closely related to the Babuška-Aziz inequality and to the corresponding domain specific constants.

Definition 2.2. The domain $\Omega \subset \mathbb{R}^{n}(n=2,3)$ supports the Babuška-Aziz inequality for the divergence if there is a positive constant $C$ depending only on the domain $\Omega$ such that for every $u \in L_{2}(\Omega)$ with $u_{\Omega}=0$ there is a $v \in H_{0}^{1}(\Omega)^{n}$ such that $\operatorname{div} v=u$ and

$$
|v|_{1}^{2} \leq C\|u\|^{2} .
$$

The least possible constant in (5) is denoted by $C_{\Omega}$ and is called the Babuška-Aziz constant for the divergence of the domain. $C_{\Omega}<\infty$ was proved for bounded Lipschitz domains in [3] and was generalized for John domains in [1].

The inequality (5) can be formulated as $|v|_{1}^{2} \leq C\|\operatorname{div} v\|^{2}$ for every function $v$ in the orthogonal complement of the kernel of the divergence in $H_{0}^{1}(\Omega)^{n}$. Similarly there is a Babuška-Aziz inequality for the rotation:

$$
|v|_{1}^{2} \leq \tilde{C}\|\operatorname{rot} v\|^{2}
$$

provided $v$ is in the orthogonal complement of the kernel of the rotation in $H_{0}^{1}(\Omega)^{3}$. According to $[6,7,21]$ there also hold

$$
C_{\Omega}=1+\Gamma_{\Omega} \text { and } \tilde{C}_{\Omega}=1+\tilde{\Gamma}_{\Omega}
$$

for any planar or spatial domain the constants being simultaneously finite or infinite.

The third class of inequalities utilized in this paper is the class of the improved Poincaré inequalities.

Definition 2.3. The domain $\Omega \subset \mathbb{R}^{n}(n=2,3)$ supports supports the improved Poincaré inequality if there is a positive constant $P$ depending only on the domain $\Omega$ and on the exponents $\alpha, p$ and $q$ such that

$$
\left\|u-u_{\Omega}\right\|_{L_{q}(\Omega)}^{q} \leq P\left\|d_{\Omega}^{\alpha} \nabla u\right\|_{L_{p}(\Omega)}^{q}
$$

holds for every $u \in L_{1, l o c}(\Omega)$ such that $d_{\Omega}^{\alpha} \nabla u \in L_{p}(\Omega)^{n}$, where $d_{\Omega}(x)=\operatorname{dist}(x, \partial \Omega)$ is the distance of $x \in \Omega$ to the boundary. 
It was proved in [4] in case $p=q$ for domains whose boundary is locally the graph of a Hölder continuous function of order $\alpha$ and it was generalized in [14] for $0 \leq \alpha \leq 1$, $p(1-\alpha)<n$ and $p \leq q \leq \frac{n p}{n-p(1-\alpha)}$ in a class of domains including John-domains.

In case $\alpha=0$ and $q=p=2$ one has the classical Poincaré inequality but in this paper we utilize the case $q=p=2$ and $\alpha=1$ in the form

$$
\left\|u-u_{\Omega}\right\|^{2} \leq P_{\Omega}\left\|d_{\Omega} \nabla u\right\|^{2}
$$

wherein the improved Poincare constant $P_{\Omega}$ of $\Omega$ is the least possible positive constant satisfying (9). For bounded simply connected planar domains it was proved in [15] that the domain supports the Babuška-Aziz inequality (5) iff it supports the improved Poincaré inequality (9) and iff $\Omega$ is a John domain. For more general domains there are additional properties needed in order to have equivalence between $\Omega$ being a John domain and $\Omega$ supporting the inequalities (5) and (9), c.f. [15].

Remark 2.4. As proved in [5] for convex domains in arbitrary dimensions the constant $P$ in (8) can be estimated from above by a scalar multiple of the product $\eta(\Omega)^{2 \alpha} \operatorname{diam}(\Omega)^{2-2 \alpha}$, where $\eta(\Omega)$ and $\operatorname{diam}(\Omega)$ denote the eccentricity and the diameter of $\Omega$, respectively. This estimator is independent of $\operatorname{diam}(\Omega)$ only for $\alpha=1$ in which case the improved Poincaré constant $P_{\Omega}$ in (9) can be estimated by a scalar multiple (depending only on the dimension $n$ ) of $\eta(\Omega)^{2}$. Hence if one wants to derive a correspondence between the diameter invariant Friedrichs-Velte constant and the improved Poincaré constants then one has to choose $\alpha=1$ in (8).

\section{Connections between the constants}

In this section, motivated by Theorem 5.1 an Theorem 5.3 in [10], we derive connections between the Friedrichs-Velte and improved Poincaré inequalities and constants.

Lemma 3.1. If the domain $\Omega \subset \mathbb{R}^{n}(n=2,3)$ supports the improved Poincare inequality (9) with the constant $P_{\Omega}$, then it also supports the Friedrichs-Velte inequality (1) with the constant

$$
\Gamma_{\Omega} \leq 4 P_{\Omega} .
$$

Proof. Let $u$ and $v$ be conjugate harmonic functions in the sense of (3) on the spatial domain $\Omega$ supporting the improved Poincaré inequality. We develop the norm on the right-hand side of (9):

$$
\begin{aligned}
\left\|d_{\Omega} \nabla u\right\|^{2} & =\int_{\Omega} d_{\Omega}^{2}|\nabla u|^{2}=\int_{\Omega} d_{\Omega}^{2} \nabla u \cdot \operatorname{rot} v=\int_{\Omega} v \cdot \operatorname{rot}\left(d_{\Omega}^{2} \nabla u\right) \\
& =\int_{\Omega} v \cdot 2 d_{\Omega} \nabla d_{\Omega} \times \nabla u=\int_{\Omega} 2 d_{\Omega} \nabla u \cdot\left(v \times \nabla d_{\Omega}\right)
\end{aligned}
$$

We estimate by the Cauchy-Schwarz inequality and we also use $\left|\nabla d_{\Omega}\right|=1$ for the boundary distance function of $\Omega$ valid a.e. in $\Omega$.

$$
\left|\int_{\Omega} 2 d_{\Omega} \nabla u \cdot\left(v \times \nabla d_{\Omega}\right)\right| \leq 2\left(\int_{\Omega}\left|d_{\Omega} \nabla u\right|^{2}\right)^{\frac{1}{2}} \cdot\left(\int_{\Omega}|v|^{2}\right)^{\frac{1}{2}} .
$$


There follows

$$
\left\|d_{\Omega} \nabla u\right\|^{2} \leq 2 \cdot\|v\| \cdot\left\|d_{\Omega} \nabla u\right\|
$$

By the improved Poincaré inequality there follows

$$
\left\|u-u_{\Omega}\right\|^{2} \leq P_{\Omega}\left\|d_{\Omega} \nabla u\right\|^{2} \leq 4 P_{\Omega}\|v\|^{2},
$$

which implies (10) for the Friedrichs-Velte constant $\Gamma_{\Omega}$ of the domain.

For planar domains the above proof remains valid with minor changes using the CauchyRiemann equations (2) instead of (3).

Remark 3.2. Theorem 5.1 in [10] formulates a similar result between the Babuška-Aziz constant for the divergence and the improved Poincaré constant for arbitrary dimensional domains, however, with another dimension dependent constant which value is not specified. This reads with the notation of Lemma 3.1

$$
\sqrt{1+\Gamma_{\Omega}} \leq c_{n}\left(1+\sqrt{P_{\Omega}}\right)
$$

Altough Lemma 3.1 is proved only for planar and spatial domains but we now have an explicit constant in the inequality estimating $\Gamma_{\Omega}$ by $P_{\Omega}$ from above.

Remark 3.3. In the proof of Lemma 3.1 we used $\left|\nabla d_{\Omega}\right|=1$ for the boundary distance function valid a.e. in $\Omega$. The proof remains valid using instead of $d_{\Omega}$ another weight function $w$ with bounded gradient on $\Omega,|\nabla w| \leq c$ and with zero boundary values on $\partial \Omega$ (appropiate solution of the eikonal equation). However, in this case one has to use another weighted Poincaré inequality instead of (8) and one has another constant in (10) instead of 4.

Remark 3.4. The constant 4 in (10) can be improved, for example for convex polygons one has more: $\Gamma_{\Omega} \leq P_{\Omega}$. In order to prove this we consider first that by [12] every convex polygon has a unique mother body (skeleton) consisting of line segments which are subsets of bisectors of angles between appropriate two sides of the polygon. The convex polygon $\Omega$ has a partition along this mother body $\Omega=\cup_{j} \Omega_{j}$. We have $\left|\nabla d_{\Omega}\right|=1$ and $\left|\Delta d_{\Omega}\right|=0$ in each subpolygon $\Omega_{j}$ hence there follows

$$
\Delta\left(d_{\Omega}^{2}\right)=2\left|\nabla d_{\Omega}\right|^{2}+2 d_{\Omega} \Delta d_{\Omega}=2
$$

for the Laplacian of the square of the boundary distance function of $\Omega$ in each subpolygon $\Omega_{j}$. On the boundary of each subpolygon $\Omega_{j}$ we have $\frac{\partial d_{\Omega}}{\partial n_{j}}>0$ because $\Omega$ is convex. By partial integration we have on each $\Omega_{j}$

$$
\int_{\Omega_{j}}\left|d_{\Omega} \nabla u\right|^{2}=\int_{\Omega_{j}} d_{\Omega}^{2} \Delta\left(\frac{1}{2} v^{2}\right)=\int_{\partial \Omega_{j}} d_{\Omega} v\left(d_{\Omega} \frac{\partial v}{\partial n_{j}}-v \frac{\partial d_{\Omega}}{\partial n_{j}}\right)+\frac{1}{2} \int_{\Omega_{j}} v^{2} \Delta\left(d_{\Omega}^{2}\right)
$$

for a conjugate harmonic pair $\nabla u=\nabla^{\perp} v$. Now summing up all these equations some boundary terms cancel out and we obtain

$$
\left\|d_{\Omega} \nabla u\right\|^{2}=\int_{\Omega} v^{2}-\sum_{j} \int_{\partial \Omega_{j}} d_{\Omega} v^{2} \frac{\partial d_{\Omega}}{\partial n_{j}}
$$


Using $\frac{\partial d_{\Omega}}{\partial n_{j}}>0$ on the boundaries of the subpolygons, there follows

$$
\left\|d_{\Omega} \nabla u\right\|^{2} \leq\|v\|^{2}
$$

which gives $\Gamma_{\Omega} \leq P_{\Omega}$.

Lemma 3.1 says that each domain which supports the improved Poincaré inequality also supports the Friedrichs-Velte inequality.

In order to prove the reverse direction we use

- the equality $C_{\Omega}=1+\Gamma_{\Omega}$ proved in [6,7] stating the simultaneous finiteness of the Friedrichs-Velte and the Babuška-Aziz constants for a domain $\Omega$ without assuming any boundary regularity and

- Theorem 5.3 in [10], in which the finiteness of the improved Poincaré constant was proved assuming the finiteness of the Babuška-Aziz constant $C_{\Omega}$ provided the domain $\Omega$ also supports a Hardy type inequality involving the boundary distance function.

Definition 3.5. The bounded domain $\Omega \subset \mathbb{R}^{n}$ supports the Hardy inequality if there is a finite positive constant $\mathrm{H}$ depending only on $\Omega$ such that

$$
\int_{\Omega} \frac{u^{2}}{d_{\Omega}^{2}} \leq H \int_{\Omega}|\nabla u|^{2}
$$

holds for every $u \in H_{0}^{1}(\Omega)$. The Hardy constant $H_{\Omega}$ of the domain is the least positive constant $H$ for which (15) holds.

Lemma 3.6. If the domain $\Omega \subset \mathbb{R}^{n}(n=2,3)$ supports the Friedrichs-Velte inequality (1) with the constant $\Gamma_{\Omega}$ and the Hardy inequality (15) with the constant $H_{\Omega}$, then it also supports the improved Poincaré inequality (8) with the constant

$$
P_{\Omega} \leq H_{\Omega}\left(1+\Gamma_{\Omega}\right)
$$

Proof. The proof is due to Durán [10], Theorem 5.3, which we reproduce here for the convenience of the reader using the notation of the present paper. Given $u \in H^{1}(\Omega)$ with zero integral mean $u_{\Omega}=0$ let $v \in H_{0}^{1}(\Omega)^{n}$ such that

$$
\operatorname{div} v=u \text { and }\|\nabla v\|^{2} \leq C_{\Omega}\|u\|^{2} .
$$

Using the Hardy inequality (15) there follows

$$
\|u\|^{2}=\int_{\Omega} u \operatorname{div} v=-\int_{\Omega} v \cdot \nabla u \leq\left\|\frac{v}{d_{\Omega}}\right\| \cdot\left\|d_{\Omega} \nabla u\right\| \leq H_{\Omega}^{\frac{1}{2}}\|\nabla v\| \cdot\left\|d_{\Omega} \nabla u\right\| .
$$

Using now the Babuška-Aziz inequality there follows

$$
\|u\|^{2} \leq H_{\Omega}^{\frac{1}{2}} C_{\Omega}^{\frac{1}{2}}\|u\| \cdot\left\|d_{\Omega} \nabla u\right\|
$$

which gives (16) utilizing $C_{\Omega}=1+\Gamma_{\Omega}$. 
Remark 3.7. Lemma 3.6 means that $H_{\Omega}<\infty$ is sufficient for an estimation of the improved Poincare constant by the Friedrichs-Velte constant. Example 4.1 in [15] shows that the validity of the Hardy inequality (15) is not necessary for such an estimation.

The proofs of Lemma 3.1 and Lemma 3.6 are applicable with minor changes for the other Friedrichs-Velte inequality (4) and for the Babuš ka-Aziz inequality (6) for the rotation.

Lemma 3.8. If the domain $\Omega \subset \mathbb{R}^{3}$ supports the Hardy inequality (15) and the FriedrichsVelte inequality (4) with the finite constants $H_{\Omega}$ and $\tilde{\Gamma}_{\Omega}$, respectively, then there is a finite positive constant $\tilde{P}_{\Omega}$ depending only on $\Omega$ such that the inequality

$$
\|v\|^{2} \leq \tilde{P} \quad\left\|d_{\Omega} \operatorname{rot} v\right\|^{2}
$$

is valid for every $v$ in the orthogonal complement of the kernel of rot in $L_{2}(\Omega)^{3}$. Moreover, we have $\tilde{P}_{\Omega} \leq H_{\Omega}\left(1+\tilde{\Gamma}_{\Omega}\right)$ for the least possible constant $\tilde{P}$ in (20).

Proof. The proof is essentially the same as that of Lemma 3.6. First use $\tilde{C}_{\Omega}=1+\tilde{\Gamma}_{\Omega}$, see [7,21]. By the Babuška-Aziz inequality for the rotation there exists $w \in H_{0}^{1}(\Omega)^{3}$ such that

$$
\operatorname{rot} w=v \text { and }\|\nabla w\|^{2} \leq \tilde{C}_{\Omega}\|v\|^{2} .
$$

There follows by the Hardy inequality (15)

$$
\|v\|^{2}=\int_{\Omega} v \operatorname{rot} w=\int_{\Omega} w \operatorname{rot} v \leq\left\|\frac{w}{d_{\Omega}}\right\| \cdot\left\|d_{\Omega} \operatorname{rot} v\right\| \leq H_{\Omega}^{\frac{1}{2}}\|\nabla w\| \cdot\left\|d_{\Omega} \operatorname{rot} v\right\| .
$$

Substituting now the Babuška-Aziz inequality for the rotation gives

$$
\|v\|^{2} \leq H_{\Omega}^{\frac{1}{2}} \tilde{C}_{\Omega}^{\frac{1}{2}}\|v\| \cdot\left\|d_{\Omega} \operatorname{rot} v\right\|
$$

from which the statement of the lemma follows.

Remark 3.9. In the improved Poincare inequality (9) the function $u-u_{\Omega}$ belongs to the orthogonal complement of the kernel of the gradient, hence (9) can be also stated as $\|u\|^{2} \leq$ $P_{\Omega}\left\|d_{\Omega} \nabla u\right\|^{2}$ for every $u \in(\operatorname{ker} \nabla)^{\perp}$. The previous Lemma 3.8 formulates an analogous inequality for the rotation instead of the gradient, hence (20) can be seen as an improved Poincare inequality for the rotation. According to [7] we have $\tilde{\Gamma}_{\Omega}<\infty$ at least for Lipschitz domains, for which the Hardy constant $H_{\Omega}$ is also finite [16], hence (20) is valid for spatial Lipschitz domains.

Remark 3.10. In two dimensions we have $\left\|d_{\Omega} \nabla u\right\|=\left\|d_{\Omega} \nabla^{\perp} u\right\|$, hence the improved Poincare constant for the vector-curl $\nabla^{\perp}$ (adjoint of the scalar rotation) coincides with the usual Poincaré constant for the gradient. For planar domains the Friedrichs-Velte constants $\Gamma_{\Omega}$ and $\widetilde{\Gamma}_{\Omega}$ are equal as well.

The counterpart of Lemma 3.1 is the following.

Lemma 3.11. If the domain $\Omega \subset \mathbb{R}^{3}$ supports the inequality (20) for every $v$ in the orthogonal complement of the kernel of the rot in $L_{2}(\Omega)^{3}$ with the least possible positive constant $\tilde{P}_{\Omega}$, then it also supports the Friedrichs-Velte inequality (4). Moreover, we have $\tilde{\Gamma}_{\Omega} \leq 4 \tilde{P}_{\Omega}$. 
Proof. The proof is similar to that of Lemma 3.1. Let $u$ and $v$ be conjugate harmonic functions on the spatial domain $\Omega$ in the sense of the Moisil-Teodorescu equations (3) normalized such that $v$ lies in the orthogonal complement of the kernel of the rot in $L_{2}(\Omega)^{3}$. We develop the norm on the right-hand side of (20) using $\left|\nabla d_{\Omega}\right|=1$ a.e. in $\Omega$.

$$
\begin{aligned}
\left\|d_{\Omega} \operatorname{rot} v\right\|^{2} & =\int_{\Omega} d_{\Omega}^{2}|\operatorname{rot} v|^{2}=\int_{\Omega} d_{\Omega}^{2} \nabla u \cdot \operatorname{rot} v=\int_{\Omega} u \cdot \operatorname{div}\left(d_{\Omega}^{2} \operatorname{rot} v\right) \\
& =\int_{\Omega} u 2 d_{\Omega} \nabla d_{\Omega} \cdot \operatorname{rot} v \leq 2\|u\| \cdot\left\|d_{\Omega} \operatorname{rot} v\right\|
\end{aligned}
$$

This implies by (20)

$$
\|v\|^{2} \leq 4 \tilde{P}_{\Omega}\|u\|^{2}
$$

which means the Friedrichs-Velte inequality (4) with $\widetilde{\Gamma}_{\Omega} \leq 4 \widetilde{P}_{\Omega}$.

Remark 3.12. Considering the similarities between the proofs of Lemma 3.6 and Lemma 3.8 and between their reversed counterparts Lemma 3.1 and Lemma 3.11 they could be possibly unified in the framework of [7] provided there is a usable version of the utilized Hardy and improved Poincaré inequalities for differential forms. However, the investigation of this is beyond the scope of the present paper.

As a consequence of these lemmata we obtain the following

Theorem 3.13. If the bounded domain $\Omega$ supports the Hardy inequality (15), then $\Omega$ supports the Friedrichs-Velte (1) and (4) and simultaneously the Babuška-Aziz inequalities (5) and (6) if and only if $\Omega$ supports the improved Poincaré inequalities (9) and (20), respectively. Moreover, we have

$$
\frac{1}{4} \Gamma_{\Omega} \leq P_{\Omega} \leq H_{\Omega}\left(1+\Gamma_{\Omega}\right) \text { and } \frac{1}{4} \tilde{\Gamma}_{\Omega} \leq \tilde{P}_{\Omega} \leq H_{\Omega}\left(1+\tilde{\Gamma}_{\Omega}\right)
$$

for the domain spacific constants in the corresponding inequalities.

Remark 3.14. Theorem 3.13 opens the possibility to obtain upper estimates for the improved Poincaré constant $P_{\Omega}$ using known exact values or estimates for the corresponding FriedrichsVelte and the Hardy constants. Such upper estimates for $\Gamma_{\Omega}$ of a star-shaped planar or spatial domain are given in $[6,13,17]$ which upper estimates depend on the eccentricity of the domain with respect to the center of the star-shapedness. As shown in [5] the Poincare constant (9) of a convex domain can be estimated by its eccentricity $\eta$, i.e. $P_{\Omega} \leq c \eta^{2}$ for some positive constant $c$. According to Theorem 6.2 in [6] and Theorem 3.13 this remains valid for a planar star-shaped domain as well because its Hardy constant is at most 16, see [2].

$$
P_{\Omega} \leq 16\left(\eta+\sqrt{\eta^{2}-1}\right)^{2} \leq 64 \eta^{2}
$$

where $\eta=\frac{R}{r}$ for the domain $\Omega$ star-shaped with respect to a disc of radius $r$ and contained in a concentric disc of radius $R$. 
Remark 3.15. As one sees the improved Poincaré inequalities imply the corresponding Friedrichs-Velte and Babuška-Aziz inequalities without any condition on the domain, the validity of the Hardy inequality is required only for the reverse implication. Finiteness of the Hardy constant was proved in [16] and [4] for Lipschitz and for Hölder domains, respectively. For upper estimations of the Hardy constant in terms of geometric characteristics of the domain c.f. [9] and the references given there.

\section{Concluding remarks}

The main result of this paper is twofold. First, we proved by esimating the corresponding domain specific constants by each other that planar and spatial domains satisfying the Hardy inequality simultaneously support the Friedrichs-Velte inequality, the Babuška-Aziz inequality for the divergence and the improved Poincare inequality for the gradient. This enables us to extend the validity of known upper estimates for the Friedrichs-Velte constants for the improved Poincare constant for the gradient. Second, in the three dimensional case we derived with the same method a novel improved Poincare inequality for the rotation.

\section{References}

[1] Acosta G., Durán R. G., Muschietti M. A., Solutions of the divergence operator on John domains, Adv. Math. 206 (2006) 373-401. doi:10.1016/j.aim.2005.09.004

[2] Ancona A., On strong barriers and an inequality of Hardy for domains in $\mathbb{R}^{n}$, J. London Math. Soc. (2) 37 (1986), 274-290. doi:10.1112/j1ms/s2-34.2.274

[3] Babuška I., Aziz A. K., Survey lectures on the mathematical foundations of the finite element method, in The Mathematical Foundations of the Finite Element Method with Applications to Partial Differential Equations (edited by A.K. Aziz), Academic Press, New York, 1972.

[4] Boas H. B., Straube E. J., Integral inequalities of Hardy and Poincare type, Proc. Amer. Math. Soc. 103 (1988), 172-176. doi:10.2307/2047547

[5] Chua S., Wheeden R. L., Weighted Poincaré inequalities on convex domains, Math. Res. Lett. 17(5) (2010), 993-1011. doi:10.4310/MRL.2010.v17.n5.a15

[6] Costabel M., Dauge M.: On the inequalities of Babuška-Aziz, Friedrichs and Horgan-Payne, Arch. Rational Mech. and Anal. 217 (2015) No 3, 873-898. doi:10.1007/s00205-015-0845-2

[7] Costabel M., Inequalities of Babuška-Aziz and Friedrichs-Velte for Differential Forms. In: Maz'ya V., Natroshvili D., Shargorodsky E., Wendland W. (eds) Recent Trends in Operator Theory and Partial Differential Equations. Operator Theory: Advances and Applications, vol 258. (2017) Birkhäuser, Cham doi:10.1007/978-3-319-47079-5_4

[8] Crouzeix M., On an operator related to the convergence of Uzawa's algorithm for the Stokes equation. In: Computational Science for the 21st Century (J. Périaux et al., eds.). New York: Wiley 1997, 242-249.

[9] Davies E. B., A review of Hardy inequalities, in: The Maz'ya Anniversary Collection, vol. 2, Oper. Theory Adv. Appl., vol 110 (Birkhäuser, Basel, 1999), 55-67. doi:10.1007/978-3-0348-8672-7_5

[10] Durán R.G., An elementary proof of the continuity from $L_{0}^{2}(\Omega)$ to $H_{0}^{1}(\Omega)^{n}$ of Bogovskii's right inverse of the divergence, Revista de la Unión Matemática Argentina 53(2) (2012), 59-78. http://inmabb.criba.edu.ar/revuma/pdf/v53n2/v53n2a06.pdf

[11] Friedrichs K., On certain inequalities and characteristic value problems for analytic functions and for functions of two variables. Trans. AMS 41 (1937), 321-364. https://www.ams.org/journals/tran/1937-04103/

[12] Gustafsson B., On mother bodies of convex polyhedra, SIAM J. Math. Anal. 29(5) (1998), 1106-1117. doi:10.1137/S0036141097317918

[13] Horgan C. O. and Payne L. E., On inequalities of Korn, Friedrichs and Babuška-Aziz. Achive Rat.Mech.Anal. 82 (1983), 165-179. doi:10.1007/BF00250935

[14] Hurri-Syrjänen R., An improved Poincaré inequality, Proc. Amer. Math. Soc. 120(1) (1994), $213-222$. doi:10.2307/2160188 
[15] Jiang R., Kauranen A., Koskela P., Solvability of the divergence equation implies John via Poincaré inequality, Nonlinear Anal. 101 (2014), 80-88. doi:10.1016/j.na.2014.01.021

[16] Nečas J., Sur une méthode pour résoudre les équations aux dérivées partielles du type elliptique, voisine de la variationnelle, Ann. Scuola Norm. Sup. Pisa (3) 16 (1962), 305-326. www.numdam.org/item?id= ASNSP_1962_3_16_4_305_0

[17] Payne L. E., A bound for the optimal constant in an inequality of Ladyzhenskaya and Solonnikov, IMA Journal of Applied Mathematics 72 (2007), 563-569. doi:10.1093/imamat/hxm028

[18] Shapiro H.S., On some Fourier and distribution-theoretic methods in approximation theory, in vol. Approximation Theory. III, Proc. Conf. held in Austin, Texas, 1980 (W. Cheney et. al., eds.), Academic Press, San Diego (1980), 87-124.

[19] Stoyan G., Towards discrete Velte decompositions and narrow bounds for inf-sup constants, Computers \& Maths. with Appls., 38, 7-8, (1999), 243-261. doi:10.1016/S0898-1221(99)00254-0

[20] Stoyan G., Iterative Stokes solvers in the harmonic Velte subspace, Computing 67 (2000), 13-33. doi:10.1007/s006070170014

[21] Velte W., On inequalities of Friedrichs and Babuška-Aziz in dimension three, Journal for Analysis and its Applications, Vol.17, No.4 (1998), 843-857. doi:10.4171/ZAA/854 